\title{
Respostas Pressóricas Pós-exercícios com Pesos Executados em Diferentes Sobrecargas por Mulheres Normotensas
}

\section{Post-exercise Pressoric Responses of Exercises Performed at Different Loads by Normotensive Women}

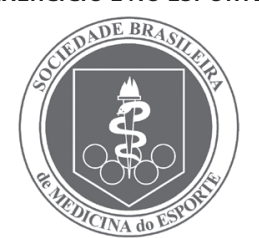

Artigo Original

\author{
André Luiz Demantova Gurjão $0^{1,2}$ \\ Emanuel Péricles Salvador1,3 \\ Edilson Serpeloni Cyrino' \\ Aline Mendes Gerage' \\ Durcelina Schiavoni' \\ Sebastião Gobbi² \\ 1. Grupo de Estudo e Pesquisa em \\ Metabolismo, Nutrição e Exercício. \\ Centro de Educação Física e \\ Esporte. Universidade Estadual de \\ Londrina. Londrina/PR. \\ 2. Laboratório de Atividade Física \\ e Envelhecimento. Departamento \\ de Educação Física. Universidade \\ Estadual Paulista. Rio Claro/SP. \\ 3. Faculdade de Saúde Pública. \\ Departamento de Nutrição. \\ Universidade de São Paulo. São \\ Paulo/SP.
}

\section{Endereço para correspondência: Edilson Serpeloni Cyrino, Grupo de Estudo e Pesquisa em Metabolismo, Nutrição e Exercício, Centro de Educação Física e Esporte, Universidade Estadual de Londrina, Rod. Celso Garcia Cid, km 380 \\ - Campus Universitário - 86051-990 \\ - Londrina, PR - Brasil. \\ E-mail: emcyrino@uel.br}

Submetido em 01/10/2007

Versão final recebida em 14/04/2008 Aceito em 27/05/2008

\begin{abstract}
RESUMO
O treinamento com pesos (TP) vem sendo amplamente recomendado para a melhoria de alguns componentes da aptidão física relacionada à saúde. Mais recentemente, vem-se discutindo o impacto do TP em indivíduos com disfunções crônico-degenerativas como a hipertensão arterial. Assim, o objetivo deste estudo foi comparar o efeito de um programa de TP executado com diferentes sobrecargas sobre o comportamento da pressão arterial (PA) pós-exercício. Para tanto, 16 mulheres jovens (20,5 \pm 1,5 anos) e aparentemente saudáveis, após ser submetidas a oito semanas de TP para equiparação dos níveis de condicionamento muscular, foram separadas, aleatoriamente, em dois grupos. A partir daí, cada grupo realizou 12 semanas de TP sob diferentes intensidades ( $\mathrm{Gl}=3 \times$ 6-8 RM e Gll = $3 \times 10-12$ RM, respectivamente), com a freqüência de três sessões semanais em dias alternados. Medidas de PA foram obtidas em repouso, por meio do método auscultatório, e nos minutos 1, 5, 10 e 30 após o término de uma sessão de TP. ANOVA two way para medidas repetidas foi utilizada nas comparações entre grupos nos diferentes períodos de tempo, ao passo que o teste post hoc de Scheffé foi empregado para a identificação das diferenças específicas, sendo adotado nível de significância de 5\%. Nenhuma diferença estatisticamente significante foi encontrada, tanto para a PA sistólica (PAS) quanto diastólica (PAD), na comparação entre os grupos. Todavia, o efeito hipotensivo do TP foi identificado somente no Gl, a PAS apresentando redução significante, quando comparada com os valores de repouso, a partir do $10^{\circ}$ minuto de recuperação pós-exercício $(P<0,05)$. Os resultados sugerem que a resposta hipotensiva pósexercício acarretada pelo TP parece ser melhorada discretamente por protocolos de maior intensidade.
\end{abstract}

Palavras-chave: exercícios com pesos, pressão arterial, saúde da mulher.

\begin{abstract}
Resistance training (RT) has been widely recommended for improvement of some health-related physical fitness components. Recently, the impact of RT in individuals with chronic-degenerative dysfunctions such as hypertension has been discussed. Thus, the aim of this study was to compare the effects of one program of resistance training performed at different intensities on post-exercise blood pressure response. Therefore, sixteen apparently healthy young women $(20.5 \pm 1.5$ year-old) were randomly separated in two groups after having been submitted to an eight-week RT for equalization of muscular fitness levels. Each group then performed 12 weeks of RT prescribed by one of two different-intensity protocols (GI =3 ×6-8 RM; GII = 3 x 10-12 RM; three alternated days per week). Systolic (SBP) and diastolic (DBP) blood pressures were assessed at rest and at minutes 1, 5, 10 and 30 after training, through auscultatory technique. Two-way ANOVA for repeated measures was used for comparison between groups at the different moments, and Scheffé post-hoc test ( $\mathrm{a}=5 \%$ ) was applied to point out where the differences occur. No significant statistical difference $(P>0.05)$ was found for SBP or DBP between groups at any moment. However, the RT hypotensive effect was observed only for Gl, with the SBP showing a significant reduction when compared to rest values at minutes 10 and 30 of recovery $(P<0.05)$. In conclusion, only higher intensity resistance training can exert a slightly acute hypotensive effect on young females.
\end{abstract}

Keywords: weight exercises, blood pressure, women's health.

\begin{abstract}
INTRODUÇÃO
O aumento dos níveis pressóricos, em situação de repouso guarda estreita relação com o desenvolvimento/agravamento de diversas disfunções crônico-degenerativas que, por sua vez, estão relacionadas à elevação das taxas de morbimortalidade, em particular, por doenças cardiovasculares $^{(1)}$. Assim, a hipertensão arterial (HA) pode ser considerada, atualmente, um dos principais problemas de saúde pública, em diversos países do mundo, atingindo um contingente cada vez maior, tanto de homens quanto de mulheres, em diferentes faixas etárias.
\end{abstract}

Dentre as principais causas para o estabelecimento do quadro hipertensivo destacam-se o reduzido nível de atividade física habitual, hábitos alimentares inadequados e o excesso de gordura corporal, sobretudo em mulheres ${ }^{(2,3)}$. Desse modo, a adoção de estratégias de intervenção que incluam mudanças no estilo de vida pode favorecer a prevenção, bem como o tratamento da HA.

Dentre as diferentes estratégias não medicamentosas que vêm sendo recomendadas pela literatura para o controle da pressão arterial (PA), destaca-se a prática regular de programas de exercícios físicos 
de diferentes naturezas. Nesse sentido, a grande maioria dos estudos disponíveis na literatura tem relatado importantes respostas hipotensivas pós-exercício (RHPE) induzidas de forma aguda, principalmente por exercícios predominantemente aeróbios ${ }^{(4)}$.

Por outro lado, o aumento das informações a cerca dos efeitos positivos do treinamento com pesos (TP) sobre as variáveis associadas à aptidão física relacionada à saúde tem motivado diferentes organizações internacionais ligadas à saúde a incluirem a prática regular sistematizada e supervisionada de exercícios com pesos em suas recomendações ${ }^{(1,5)}$.

Todavia, quando o foco passa a ser a prevenção ou tratamento da HA, as mesmas organizações têm indicado o TP apenas como uma atividade adicional à prática de exercícios predominantemente aeróbios. Esse fato parece plenamente justificável pela carência de informações mais consistentes sobre o possível efeito hipotensivo associado à prática de exercícios com pesos ${ }^{(1)}$, visto que estudos que procuraram investigar as respostas pressóricas acarretadas pela prática do TP, até o presente momento, têm relatado desde nenhuma modificação significante ${ }^{(6)}$, até modificações positivas ou negativas, tanto na PA sistólica (PAS) quanto na PA diastólica (PAD) ${ }^{(7,8)}$.

Vale ressaltar que grande parte das dificuldades em interpretar os resultados de vários estudos disponíveis na literatura envolvendo o TP e a PA parece estar relacionada à falta de controle dos níveis de aptidão física prévia dos sujeitos investigados ${ }^{(9)}$, uma vez que modificações associadas à prática de exercícios com pesos parecem ser bastante diferenciadas entre praticantes iniciantes e experientes ${ }^{(10)}$, e aos protocolos experimentais adotados, que na maioria das vezes têm sido estruturados de forma muito diferenciada com relação ao volume (número de exercícios, séries e repetições, freqüência semanal) e à intensidade (carga, velocidade de execução dos movimentos, intervalos de recuperação entre as séries e os exercícios) ${ }^{(11,12)}$.

Com base nas informações apresentadas anteriormente, desvendar as possíveis razões para resultados tão contrastantes parece ser uma tarefa relativamente complexa, visto que a simples manipulação de variáveis que envolvem a prescrição do TP (volume e intensidade de esforço) pode desencadear respostas bastante diferenciadas.

Considerando que a redução da pressão arterial em repouso, mesmo em indivíduos normotensos, apresenta importante impacto na redução do risco para o desenvolvimento de doenças cardiovasculares ${ }^{(1)}$, o objetivo deste estudo foi comparar o efeito de um programa de TP executado com diferentes sobrecargas sobre as RHPE em mulheres normotensas.

\section{MÉTODOS}

\section{Sujeitos}

Vinte universitárias foram selecionadas para participar deste estudo. Como critérios iniciais de inclusão, as participantes deveriam ser normotensas e não deveriam praticar atividade física regular e sistematizada mais do que uma vez por semana, bem como deveriam não ter participado regularmente de nenhum programa de TP ao longo dos últimos seis meses precedentes ao início do experimento. Todas participantes responderam a uma anamnese especificamente desenvolvida para investigar os aspectos de saúde das voluntárias e nenhuma disfunção metabólica ou musculoesquelética foi relatada.

Quatro mulheres não concluíram o experimento por diferentes razões, incluindo abandono voluntário, não-cumprimento de procedimentos estabelecidos previamente ao início do estudo ou problemas particulares. Assim, 16 mulheres compuseram a amostra que finalizou o experimento.

Todas as participantes, após receber informações sobre as finalidades do estudo e os procedimentos aos quais seriam submetidas, assinaram um termo de consentimento livre e esclarecido. O estudo foi aprovado pelo Comitê de Ética em Pesquisa da Universidade Estadual de Londrina, de acordo com as normas da Resolução 196/96 do Conselho Nacional de Saúde sobre pesquisa envolvendo seres humanos.

\section{Antropometria}

A massa corporal foi mensurada em uma balança de leitura digital, da marca Urano, modelo PS 180A, com precisão de 100g e carga máxima de $180 \mathrm{~kg}$, ao passo que a estatura foi determinada em um estadiômetro de madeira com precisão de $1 \mathrm{~mm}$, de acordo com os procedimentos recomendados por Gordon et al. ${ }^{(13)}$. A partir dessas medidas foi calculado o índice de massa corporal (IMC), por meio da relação entre a massa corporal e o quadrado da estatura, sendo a massa corporal expressa em quilogramas (kg) e a estatura em metros (m).

\section{Programa de treinamento com pesos}

O protocolo de TP foi dividido em duas etapas. As primeiras oito semanas (etapa 1) foram destinadas ao período de adaptação à prática do treinamento e também à aprendizagem dos gestos motores. O protocolo de treinamento nessa etapa foi idêntico para todas as participantes, sendo adotado um exercício para cada grupo muscular que foi realizado em três séries de 12-15 repetições máximas (RM). Após o término das oito semanas iniciais, as participantes foram divididas aleatoriamente em dois grupos, que executaram o mesmo programa de exercícios sob diferentes intensidades $(\mathrm{Gl}=3 \times 6-8 \mathrm{RM})$ e $(\mathrm{Gll}=$ $3 \times 10-12 \mathrm{RM}$ ), por um período de 12 semanas consecutivas (etapa 2). O programa de TP foi executado com freqüência semanal de três sessões, em ambas as etapas. Somente os indivíduos que participaram de pelo menos $85 \%$ das sessões de TP previstas, tanto para a primeira quanto para a segunda etapa (20 sessões e 31 sessões na etapa 1 e 2, respectivamente), fizeram parte da amostra analisada.

Os exercícios que compuseram o programa de TP foram executados na seguinte ordem: voador e supino em banco horizontal (peitoral); puxada pela frente e remada baixa (costas); elevação lateral (ombros); rosca direta (bíceps) e tríceps pulley (tríceps); mesa flexora e leg press (coxas); panturrilha no leg press (pernas) e flexões abdominais. Vale ressaltar que os exercícios abdominais foram realizados em três séries de 30-50 repetições, sem sobrecarga adicional, em virtude de a sala de treinamento não possuir aparelho específico para a realização da flexão abdominal. Portanto, optou-se pela manutenção do exercício de flexão abdominal no solo, sem sobrecarga adicional (apenas o próprio peso corporal) e um número maior de repetições em relação ao protocolo utilizado para os outros exercícios.

Cada exercício foi executado em três séries, sendo adotado o método de cargas fixas. As cargas utilizadas foram compatíveis com o número de repetições máximas estipuladas para cada exercício. Os indivíduos receberam orientação para que as cargas de treinamento fossem reajustadas sempre que o número máximo de repetições preestabelecidas para cada exercício fosse atingido em todas as séries, na tentativa de que a intensidade inicial pudesse ser preservada.

Tanto as cargas iniciais quanto os reajustes periódicos, nas cargas utilizadas nos diferentes exercícios, foram estabelecidos com base nos resultados obtidos mediante a aplicação de testes de peso por repetições máximas ${ }^{(14)}$. Vale ressaltar que, em ambas as etapas, o intervalo de recuperação estabelecido entre as séries, em cada exercício, foi de 60 a 90s e, entre os exercícios, de dois a três minutos.

Embora a velocidade de execução não tenha sido controlada, os sujeitos foram orientados a executar a fase concêntrica mais rapidamente (um a dois segundos) do que a fase excêntrica (dois a quatro segundos). As participantes foram orientadas, ainda, a não realizar outro tipo de atividade física regular sistematizada durante o período de duração do estudo, de modo que o impacto do TP pudesse ser avaliado de forma isolada.

\section{Pressão arterial}

A mensuração da PA foi realizada na última semana da segunda etapa de treinamento, antes e após uma única sessão de TP em am- 
bos os grupos. Para tanto, foi empregado o método auscultatório, com auxílio de um esfigmomanômetro aneróide ( $A L P^{\circledR}$, Japão) e um estetoscópio (Rappaport ${ }^{\circledR}$, Brasil).

A determinação da PA em repouso foi obtida com os sujeitos permanecendo na posição sentada por um tempo não inferior a 10 min, no local onde seria realizada a sessão de TP. Essa medida foi utilizada como referência para as comparações com os valores obtidos no período de recuperação pós-exercício. A primeira e a quarta fases dos sons de Korotkov foram adotadas como referência para a determinação da PAS e da $P A D$, respectivamente. Posteriormente à determinação da PA em repouso, iniciava-se a sessão de TP. Ao final da sessão, as participantes permaneceram sentadas em uma cadeira, em repouso, e a PAS e a PAD foram medidas nos minutos 1, 5, 10 e 30. Nesse período foram estabelecidos somente diálogos essenciais e não foi permitido que as participantes ingerissem qualquer tipo de bebida ou alimento. Todas as medidas foram realizadas por um único avaliador com experiência prévia nesse tipo de medida. O erro técnico de medida para a PAS e PAD foi de 3,5 e 3,0 $\mathrm{mmHg}$, respectivamente, ao passo que o coeficiente de correlação intraclasse para a PAS foi de $0,98(95 \%$ IC; 0,89 - 0,99) e para a PAD foi de 0,96 (95\% IC; 0,80 - 099).

Vale ressaltar que a verificação da calibração do manômetro aneróide foi realizada por meio de um manômetro de coluna de mercúrio seguindo as recomendações de Pickering et al.(15).

\section{Tratamento estatístico}

O teste de Shapiro-Wilk foi utilizado, inicialmente, para análise da distribuição dos dados. Após a confirmação da normalidade dos dados as características físicas iniciais dos dois grupos (Gl e GII) foram comparadas pelo teste $t$ de Student para amostras independentes com número de elementos diferentes. Análise de variância (ANOVA) 2 X 5 para medidas repetidas foi empregada para as comparações entre os grupos (Gl e Gll) e os momentos (repouso, 1, 5, 10 e 30 min pósexercício). O teste post hoc de Scheffé, para comparações múltiplas, foi empregado para a identificação das diferenças específicas nas variáveis em que os valores de $F$ encontrados foram superiores aos do critério de significância estatística estabelecido $(P<0,05)$. As informações foram processadas no pacote computacional Statistica ${ }^{T M}$, versão 5.1.

\section{RESULTADOS}

A tabela 1 apresenta informações sobre idade, massa corporal, estatura e índice de massa corporal (IMC) das mulheres investigadas. Diferenças estatisticamente significantes foram encontradas entre os grupos $(\mathrm{Gl}>\mathrm{Gll})$ apenas na estatura $(\sim 7 \mathrm{~cm})$ e na massa corporal $(\sim 7 \mathrm{~kg})$.

$\mathrm{Na}$ tabela 2 encontram-se os resultados referentes à PA em repouso e no período de 1, 5, 10 e 30 min após o esforço, em ambos os grupos. Nenhuma diferença estatisticamente significante foi verificada para a PAS e PAD, em repouso, quando comparados os grupos Gl e Gll. Comparando-se os valores de repouso com aqueles encontrados no pós-exercício, verificaram-se diferenças estatisticamente significantes

Tabela 1. Características gerais dos grupos submetidos ao treinamento com pesos de alta intensidade (GI) e de intensidade moderada (GII)

\begin{tabular}{l|c|c|c}
\hline \multicolumn{1}{c|}{ Variáveis } & Gl $(n=9)$ & GII $(n=7)$ & $P$ \\
\hline Idade (anos) & $22,2 \pm 1,6$ & $20,6 \pm 1,4$ & 0,66 \\
\hline Massa corporal $(\mathrm{kg})$ & $60,7 \pm 5,8$ & $54,1 \pm 6,5$ & 0,05 \\
\hline Estatura $(\mathrm{cm})$ & $170,0 \pm 0,1$ & $163,0 \pm 0,1$ & 0,02 \\
\hline IMC $\left(\mathrm{kg} / \mathrm{m}^{2}\right)$ & $21,0 \pm 2,2$ & $20,4 \pm 2,3$ & 0,65 \\
\hline
\end{tabular}

Nota. Os resultados estão expressos em valores médios ( \pm DP). IMC = índice de massa corporal. para a PAS, a partir do $10^{\circ}$ minuto de recuperação, somente no grupo GI $(\sim 9 \mathrm{mmHg}$; $P<0,05)$. Com relação aos valores da PAD, não foram encontradas diferenças significantes entre os valores obtidos em repouso e pós-exercício, em ambos os grupos. Embora o efeito isolado do tempo tenha sido observado para a PAS $(P<0,01)$, não foi verificada interação significativa grupo $X$ tempo $(P>0,05)$. Assim, nenhum efeito que pudesse ser atribuído às diferentes intensidades de treinamento sobre as respostas hipotensivas foi identificado.

Tabela 2. Comportamento da pressão arterial sistólica (PAS) e diastólica (PAD) em repouso e após sessão de treinamento com pesos de alta intensidade (GI) e de intensidade moderada (GII)

\begin{tabular}{l|c|c|l|c|c}
\hline \multicolumn{1}{c|}{ Variáveis } & GI (n=9) & GII (n= 7) & Efeitos & F & P \\
\hline \multicolumn{7}{|l|}{ PAS (mmHg) } \\
\hline Repouso & $112,0 \pm 5,0$ & $110,0 \pm 6,3$ & ANOVA & & \\
\hline 1 minuto & $118,0 \pm 4,5$ & $116,3 \pm 3,5$ & & & \\
\hline 5 minutos & $106,4 \pm 6,5$ & $107,4 \pm 2,8$ & Grupo & $<0,01$ & 0,98 \\
\hline 10 minutos & $103,1 \pm 7,0^{*}$ & $104,3 \pm 3,5$ & Tempo & 37,77 & $<0,01$ \\
\hline 30 minutos & $102,9 \pm 8,0^{*}$ & $104,9 \pm 4,4$ & Grupo X tempo & 0,97 & 0,43 \\
\hline
\end{tabular}

\section{PAD (mmHg)}

\begin{tabular}{l|l|l|l|l|l}
\hline Repouso & $74,9 \pm 8,5$ & $72,9 \pm 4,4$ & ANOVA & & \\
\hline 1 minuto & $77,3 \pm 9,2$ & $71,8 \pm 6,3$ & & & \\
\hline 5 minutos & $74,7 \pm 8,0$ & $72,0 \pm 4,6$ & Grupo & 0,75 & 0,40 \\
\hline 10 minutos & $74,7 \pm 9,0$ & $72,3 \pm 6,1$ & Tempo & 0,35 & 0,84 \\
\hline 30 minutos & $73,1 \pm 8,9$ & $72,3 \pm 3,8$ & Grupo $X$ tempo & 0,60 & 0,66 \\
\hline
\end{tabular}

${ }^{*} P<0,05$ vs. Repouso.

Nota. Os resultados estão expressos em valores médios ( \pm DP).

A figura 1 apresenta o comportamento da pressão arterial média (PAM) no repouso e durante o período de acompanhamento pósexercício (1, 5, 10 e $30 \mathrm{~min})$. A falta de interação significante grupo $X$ tempo indica que a PAM apresentou comportamento relativamente semelhante nos grupos Gl e Gll, nos períodos subseqüentes ao final da sessão de exercícios com pesos. Apenas efeito isolado do tempo foi verificado para o comportamento da PAM $(P<0,01)$.

\section{DISCUSSÃO}

Diversos estudos na literatura têm investigado as respostas da PA após a realização de programas de exercícios físicos, em diferentes populações. Considerando que a população masculina tem sido alvo da maioria desses estudos e que a grande maioria das investigações tem utilizado protocolos de exercícios físicos predominantemente aeróbios, o presente estudo tentou ampliar essa abordagem investigando as respostas da PA após uma sessão de TP realizada sob duas diferentes sobrecargas, em mulheres jovens normotensas.

Embora não tenham sido identificadas interações grupo vs. tempo $(P>0,05)$, as participantes treinadas com maior intensidade (GI) apresentaram queda mais expressiva dos níveis pressóricos, alcançando diferenças significativas $(P<0,05)$ a partir do $10^{\circ}$ minuto.

Alguns estudos disponíveis na literatura têm demonstrado o importante papel da intensidade do treinamento sobre as RHPE, sobretudo em exercícios predominantemente aeróbios ${ }^{(16)}$. De forma similar ao verificado nesses tipos de exercícios físicos, as RHPE no presente estudo foram mais prolongadas e de maior magnitude quando o programa de exercícios com pesos foi executado sob maior intensidade (Gl). 


\section{COMPORTAMENTO DA PAM}

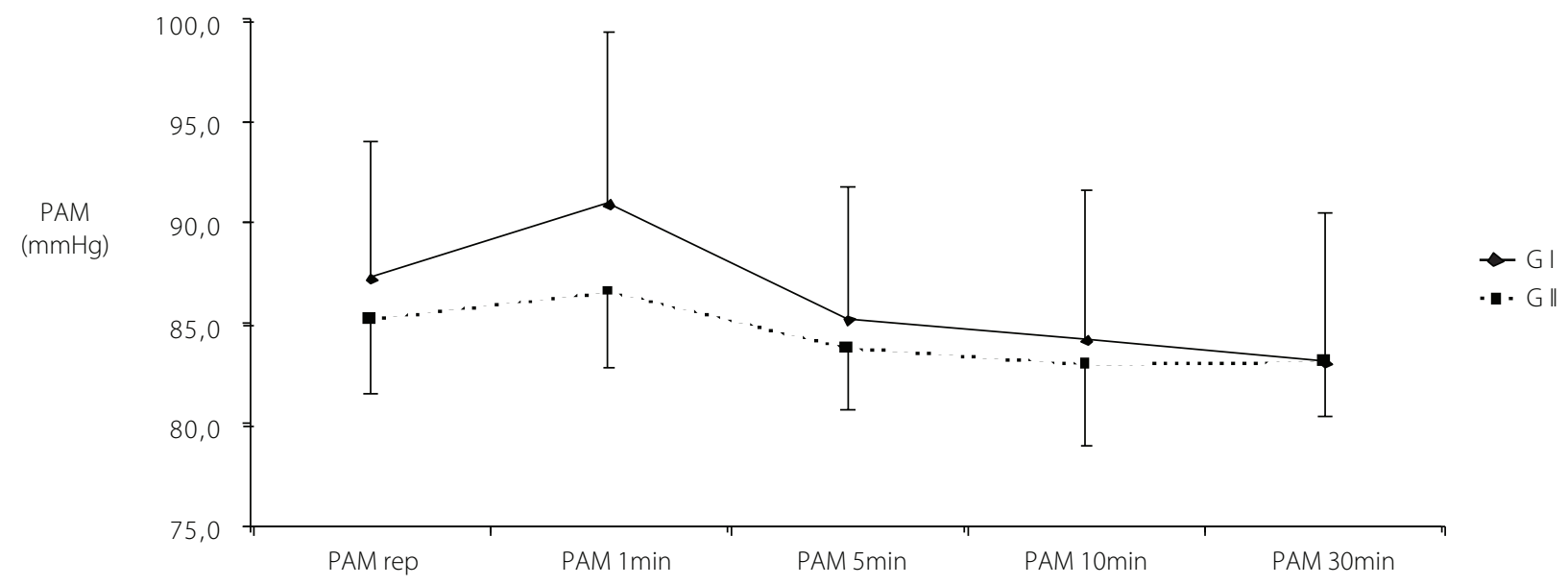

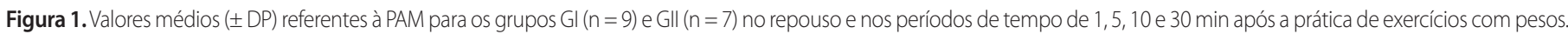
Nota. Nenhuma diferença estatisticamente significante foi encontrada intra e inter-grupos $(P>0,05)$.

Anteriormente, Polito et al.(17) haviam identificado respostas hipotensivas após duas sessões de TP realizadas em diferentes intensidades, mantendo a mesma carga de trabalho em ambos os protocolos (sessão $1=3$ X 6-RM e sessão $2=3$ X 12-RM a 50\% de 6-RM). Diferente do verificado no presente estudo, que encontrou diferenças somente para o grupo $\mathrm{Gl}$, os autores encontraram diferenças estatisticamente significantes para ambas as intensidades investigadas $(P<0,05)$. Entretanto, quando os mesmos indivíduos foram submetidos a esforços mais intensos, as respostas hipotensivas, além de mais prolongadas foram, também, de maior magnitude. Os achados deste estudo corroboram em parte esses resultados. Ainda que não tenham sido identificadas diferenças estatisticamente significantes, uma tendência para respostas hipotensivas também foi verificada para o grupo $\mathrm{Gll}(P=0,07)$.

Em contrapartida, Lizardo e Simões ${ }^{(18)}$, ao submeter 11 indivíduos normotensos com experiência prévia no TP a dois protocolos de diferentes intensidades (30 e 80\% de 1-RM), observaram que, apesar de as duas intensidades de exercício resultarem em RHPE para a PAS, a sessão de $30 \%$ exerceu maior efeito hipotensor, queda na PAD e diminuição mais duradoura na PAM.

Na tentativa de analisar o comportamento da PA após uma sessão de exercícios com pesos, Fisher ${ }^{(19)}$ submeteu tanto mulheres normotensas ( $n=9$ ) quanto hipertensas ( $n=7$ ), com pouca experiência de treinamento, a um programa em forma de circuito, de moderada intensidade (15 repetições a 50\% de 1-RM, em cinco exercícios). RHPE discretas foram observadas apenas para a PAS $(-2,3 \mathrm{mmHg})$, com comportamento similar sendo verificado em ambos os grupos.

No entanto, Focht e Koltyn ${ }^{(20)}$, ao investigar a influência de quatro exercícios com pesos realizados em duas diferentes intensidades (50\% e $80 \%$ de 1-RM) sobre a recuperação da PA em 84 indivíduos, não observaram RHPE durante um período de 180 min de acompanhamento, em nenhuma das intensidades utilizadas.

Um indicativo de que o volume de treinamento pode influenciar as RHPE foi descrito por Mediano et al.(21) ao investigarem o efeito da manipulação do volume em exercícios com pesos sobre o comportamento pressórico pós-esforço em 20 hipertensos controlados, submetidos a quatro diferentes exercícios. A manipulação do volume foi realizada por meio do incremento do número de séries em cada exercício da sessão (uma e três séries), mantendo-se o número de repetições fixo. Similarmente ao que tem sido relatado em esforços predominantemente aeróbios, as RHPE acarretadas por exercícios com pesos também parecem sofrer influência do volume de treinamento. De fato, a sessão de exercícios com maior volume (três séries) mostrou-se mais efetiva para a redução aguda dos níveis pressóricos quando comparada com a sessão realizada em série única.

Portanto, tanto em exercícios aeróbios quanto em exercícios com pesos, parece clara a existência da relação entre intensidade e volume de treinamento com o comportamento das RHPE. Contudo, em exercícios com pesos, as inúmeras possibilidades de combinações entre volume e intensidade tornam difícil a interpretação da melhor relação volume/intensidade de treinamento que resultará em melhores RHPE(19).

O'Connor et al. ${ }^{(8)}$ acompanharam por 120 min as respostas da PA de 14 universitárias após sessões de TP realizadas em três diferentes intensidades (40\%; 60\% e 80\% de 10-RM). Os resultados mostraram elevações estatisticamente significantes para PAS após 1 min (60\% de 10-RM) e após 1 e 15 min quando os indivíduos realizavam os esforços a $80 \%$ de 10-RM. Esse comportamento (aumento da PAS no minuto 1) também foi verificado por Mediano et al.(21), bem como no presente estudo, em valores absolutos, sem significância estatística. Acredita-se que esse fenômeno possa ser explicado, em parte, pela ativação dos quimiorreceptores em resposta à presença de metabólitos gerados pela contração muscular, ainda não completamente removidos, uma vez que Williamson et al. ${ }^{(22)}$ demonstraram a participação dos quimiorreceptores nas respostas da PA, por meio da oclusão do fluxo sanguíneo, após a realização de contrações musculares voluntárias.

Outro fator que não pode ser desprezado, na tentativa de melhor compreender os achados conflitantes verificados nos diferentes estudos, é a falta de controle dos níveis de aptidão física e a experiência prévia dos sujeitos investigados com a prática de TP. Embora alguns estudos tenham adotado em seus delineamentos o tempo de experiência em TP dos sujeitos como critério de inclusão, pode-se especular que em um mesmo estudo, haja sujeitos com tempo de experiência similar, porém, com diferentes níveis de aptidão física, o que pode comprometer a interpretação dos dados.

Nesse sentido, Brown et al. ${ }^{(23)}$ investigaram os efeitos de exercícios com pesos realizados em duas diferentes intensidades (40 e 80\% de 1-RM) na recuperação da PAS e PAD pós-esforço. Após um período de 60 min não foram encontradas RHPE significantes. De qualquer modo, dos sete voluntários participantes desse estudo (cinco homens e dois mulheres), as mulheres não possuíam qualquer experiência prévia em TP, ao passo que os homens tinham ao menos seis meses 
de experiência sem fazer referência às características dos programas de treinamento adotados, indicando que o nível de aptidão física dos sujeitos não foi controlado.

Portanto, na tentativa de minimizar as diferenças nos níveis de aptidão física das participantes do presente estudo, foi realizada uma etapa de treinamento pré-experimento com duração de oito semanas, idêntica para ambos os grupos. Após essa etapa, as participantes foram separadas aleatoriamente em dois grupos, executando o programa de exercícios sob diferentes sobrecargas por 12 semanas, com o objetivo de aproximar o nível de treinamento dos sujeitos. Dessa forma, na sessão de treinamento onde foram realizadas as mensurações da PA, as participantes possuíam o mesmo tempo de experiência (20 semanas) e similares níveis de aptidão física dentro de cada grupo. Logo, possíveis interferências nas repostas hipotensivas relativas a diferenças no nível de condicionamento entre as participantes podem não ter ocorrido.

Nossos resultados mostram que o grupo Gl apresentou RHPE estatisticamente significativas mesmo após 20 semanas de treinamento (12 semanas com alta intensidade). Embora a magnitude das respostas do grupo Gll não tenha sido suficiente para observar reduções pressóricas significativas, esse grupo apresentou comportamento semelhante ao verificado para o grupo $\mathrm{Gl}$.

Diferentes mecanismos têm sido propostos na tentativa de explicar as RHPE. Embora existam muitos fatores que podem influenciar esse comportamento e que, portanto, merecem ser mais bem investigados, acredita-se que tais mecanismos sejam similares tanto para o exercício predominantemente aeróbio quanto para o TP(24).

O decréscimo agudo da PAS após a prática de diferentes formas de exercícios parece estar mais atrelado à redução da resistência vascular periférica do que às alterações no débito cardíaco. Desse modo, as modificações na resistência vascular periférica, verificadas após esforços de diferentes naturezas, podem ser mediadas pela inibição simpática, modificação no ponto de operação do barorreflexo arterial e responsividade vascular (liberação de substâncias vasodilatadoras como o óxido nítrico) ${ }^{(1)}$.

Além disso, programas de exercícios físicos realizados com a participação de um grande número de grupamentos musculares, com intensidade elevada, podem contribuir para o decréscimo da resistência vascular periférica total. De qualquer forma, é importante salientar que existem evidências de que as RHPE podem ocorrer por diferentes vias e que a influência das alterações no débito cardíaco, para o desencadeamento dessas respostas, pode ser dependente do nível de aptidão física do sujeito ${ }^{(4)}$. No entanto, a presente investigação não avaliou tais variáveis, o que dificulta a compreensão dos mecanismos responsáveis pelas respostas observadas.

Um importante fator que não pode ser desprezado foi a falta de controle do ciclo menstrual das participantes no presente estudo, uma vez que Esformes et al. ${ }^{(25)}$, ao investigar a influência da fase do ciclo menstrual sobre o tempo e a magnitude da hipotensão após a realização de exercício predominantemente aeróbio, verificaram que as maiores concentrações de hormônios na fase ovulatória e lútea podem contribuir para atenuação das RHPE. No entanto, são necessários mais estudos que investiguem tal temática.

Vale ressaltar que, apesar de a monitoração ambulatorial da pressão arterial (MAPA) ser considerada o método mais indicado para a verificação das RHPE, sobretudo pela possibilidade de acompanhamento por longos períodos de tempo, o método auscultatório ainda tem sido amplamente utilizado para as medidas de PA, especialmente na situação de repouso, por se tratar de uma técnica de fácil aplicabilidade e baixo custo operacional.

\section{CONCLUSÃO}

Os resultados do presente estudo sugerem que, aparentemente, não existem diferenças significativas no comportamento da PA em mulheres normotensas, após exercícios com pesos realizados sob diferentes sobrecargas, embora esforços de maior intensidade tenham apresentado RHPE de maior magnitude, em valores absolutos.

\section{AGRADECIMENTOS}

Os autores agradecem ao CNPq e à Capes pelas bolsas outorgadas e a Raquel Gonçalves por seu auxílio na revisão do manuscrito.

Todos os autores declararam não haver qualquer potencial conflito de interesses referente a este artigo.

\section{REFERÊNCIAS BIBLIOGRÁFICAS}

1. Pescatello LS, Franklin BA, Fagard R, Farquhar WB, Kelley GA, Ray CA. American College of Sports Medicine position stand. Exercise and hypertension. Med Sci Sports Exerc. 2004;36(3):533-53. Review.

2. Carneiro G, Faria AN, Ribeiro Filho FF, Guimarães A, Lerário D, Ferreira SR, et al. Influência da distribuição da gordura corporal sobre a prevalência de hipertensão arterial e outros fatores de riscos em indivíduos obesos. Rev Assoc Med Brasil. 2003:49(3):306-11.

3. Hagberg JM, Park JJ, Brown MD. The role of exercise training in treatment of hypertension: an update Sports Med. 2000;30(3):193-206. Review.

4. Senitko AN, Charkoudian N, Halliwill JR. Influence of endurance exercise training status and gender on postexercise hypotension. J Appl Physiol. 2002;92(6):2368-74.

5. Fletcher GF, Balady GJ, Amsterdam EA, Chaitman B, Eckel R, Fleg J, et al. Exercise standards for testing and training: a statement for healthcare professionals from the American Heart Association. Circulation. 2001;104(14):1694-740.

6. Roltsch MH, Mendez T, Wilund KR, Hagberg JM. Acute resistive exercise does not affect ambulatory blood pressure in young men and women. Med Sci Sports Exerc. 2001;33(6):881-6.

7. Hill WD, Collins MA, DeMello JJ. Blood pressure after weight training exercise. J Appl Sport Sci Res. 1989;3:44-7.

8. O'Connor PJ, Bryant CX, Veltri JP, Gebhardt SM. State anxiety and ambulatory blood pressure following resistance exercise in females. Med Sci Sports Exerc. 1993;25(4):516-21.

9. Dujic Z, Ivancev V, Valic Z, Bakovic D, Marinovic-Terzic I, Eterovic D, et al. Postexercise hypotension in moderately trained athletes after maximal exercise. Med Sci Sports Exerc. 2006;38(2):318-22.

10. Kraemer WJ, Ratamess NA. Fundamentals of resistance training: progression and exercise prescription. Med Sci Sports Exerc. 2004;36(4):674-88. Review.

11. Polito MD, Farinatti PTV. Comparação da pressão arterial após exercícios contra-resistência: uma revisão sistemática sobre variáveis determinantes e possíveis mecanismos. Rev Bras Med Esporte. 2006;12:386-92.

12. Cornelissen VA, Fagard RH. Effect of resistance training on resting blood pressure: a meta-analysis of randomized controlled trials. J Hypertens. 2005;23(2):251-9.

13. Gordon CC, Chumlea WC, Roche AF. Stature, recumbent length, and weight. In: Lohman TG, Roche AF,
Martorell R, editors. Anthropometric standardization reference manual. Champaign: Human Kinetics Books, 1988;3-8.

14. Rodrigues CEC, Rocha PECP. Musculação: teoria e prática. Rio de Janeiro: Sprint, 1985.

15. Pickering TG, Hall JE, Appel LJ, Falkner BE, Graves J, Hill MN, et al. Recommendations for blood pressure measurement in humans and experimental animals: part 1: blood pressure measurement in humans: a statement for professionals from the Subcommittee of Professional and Public Education of the American Heart Association Council on High Blood Pressure Research. Circulation. 2005;111(5):697-716.

16. Forjaz CL, Cardoso CG Jr, Rezk CC, Santaella DF, TinucciT. Postexercise hypotension and hemodynamics: the role of exercise intensity. J Sports Med Phys Fitness. 2004;44(1):54-62.

17. Polito MD, Simão R, Senna GW, Farinatti PTV. Efeito hipotensivo do exercício de força realizado em intensidades diferentes e mesmo volume de trabalho. Rev Bras Med Esporte. 2003;9:74-7.

18. Lizardo LHF, Simões HG. Efeitos de diferentes sessões de exercícios resistidos sobre a hipotensão pós-exercícios. Rev Bras Fisioter. 2005;3:249-55.

19. Fisher MM. The effect of resistance exercise on recovery blood pressure in normotensive and borderline hypertensive women. J Strength Cond Res. 2001;15(2):210-6.

20. Focht BC, Koltyn KF. Influence of resistance exercise of different intensities on state anxiety and blood pressure. Med Sci Sport Exerc. 1999;31(3):456-63. Erratum in: Med Sci Sports Exerc. 2000;32(2):549.

21. Mediano MFF, Paravidino V, Simão R, Pontes FL, Polito MD. Comportamento subagudo da pressão arterial após o treinamento de força em hipertensos controlados. Rev Bras Med Esporte. 2005;11:337-40.

22. Williamson JW, McColl R, Mathews D, Mitchell JH, Raven PB, Morgan WP. Evidence for central command activation of the human insular cortex during exercise. J Appl Physiol. 2003;94(5):1726-34.

23. Brown SP, Clemons JM, He Q, Liu S. Effects of resistance exercise and cycling on recovery blood pressure. J Sports Sci. 1994;12(5):463-8.

24. MacDonald JR. Potential causes, mechanisms, and implications of post exercise hypotension. J Hum Hypertens. 2002;16(4):225-36. Review.

25. Esformes Jl, Norman F, Sigley J, Birch KM. The influence of menstrual cycle phase upon postexercise hypotension. Med Sci Sports Exerc. 2006;38(3):484-91. 